\title{
Application of ancillary testing in the diagnosis of hydatidiform mole: A comparison of genotyping and ploidy

\section{Sandra Wessman ${ }^{1}$, Ulrika Joneborg ${ }^{2}$, Ann Ohlsson ${ }^{1}$, Barbara Gürtl- Lackner $^{1}$, Joseph Carlson ${ }^{1}$}

${ }^{1}$ Department of Oncology-Pathology, Karolinska Institutet, and Department of Pathology and Cytology, Karolinska University Hospital, SE-171 76 Stockholm, Sweden.

2Department of Women's and Children's Health, Karolinska Institutet, and Department of Gynacologic Oncology, Karolinska University Hospital, SE-171 76 Stockholm, Sweden.

\section{Background:}

- Diagnosis of hydatidiform mole is improved through the use of ancillary diagnostic methods such as ploidy or genotyping.

- There is still discussion regarding the need for, and selection of, ancillary methods.

- Purpose: To evaluate the performance of two commonly used ancillary methods, ploidy and STR genotyping.

Methods:

- 72 POCs with suspicion of mola hydatidosa. Both ploidy and genotyping attempted.

- Final diagnosis: Combination of histology, p57 immunohistochemistry, ploidy and genotyping.

- Ploidy analysis: Image cytometry AHRENS ICM Cytometry System (Medtechnische Beratung, Hamburg, Germany).

- STR genotyping: DNA from microdissected maternal and fetal tissue. Analyzed using AmpFISTR Identifiler (Applied Biosystems, Foster City, USA), followed by quantitative capillary electrophoresis, and interpretation.

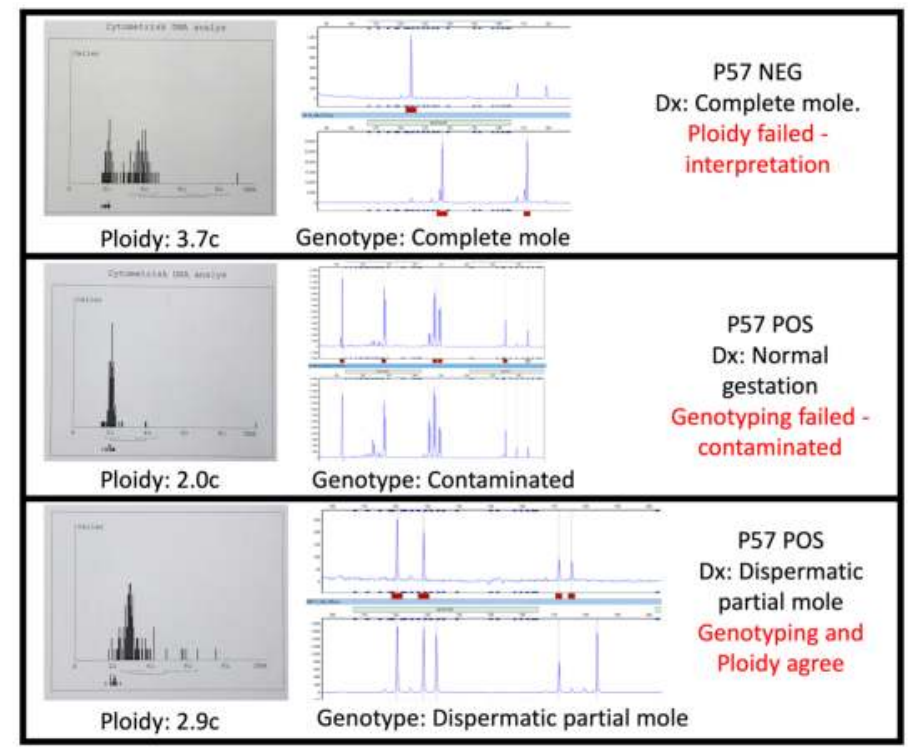

Figure 1. Representative case examples.

(Upper): PLOIDY FAILURE. Biphasic ploidy curve. Paternal only genotyping. Mix of diploid and tetraploid cells.

(Middle): GENOTYPING FAILURE: The genotyping is contaminated. (Lower box): Consistent results in all analyses.

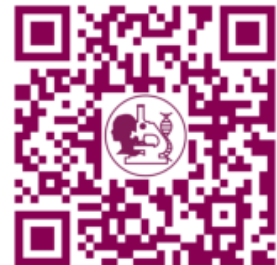

The Carlson Lab

http://www.TheCarlsonLab.se

@joseph_carlson

Molecular Gyn Cancer Research

Karolinska Institutet

Stockholm, Sweden
Results:

- Final diagnosis of the 72 cases: 42 partial moles (PM), 14 complete moles (CM) and 14 non-molar gestations.

- Ploidy analysis was successful in 50/72 cases $(70 \%)$ and genotyping was successful in 60/72 cases (83\%).

- The most common reason for failure in ploidy analysis was difficulty in interpreting the generated ploidy curves.

- The most common reason for failure in genotyping was mixing of maternal and villous material for analysis.

- Of the 22 cases where ploidy failed, there were $12 \mathrm{PM}, 7 \mathrm{CM}$, 2 non-molar gestations, and one mole of unclear type.

- Of the 12 cases where genotyping failed, there were 4 PM, one complete mole, 5 non-molar gestations and 2 moles of unclear type.

- Both ancillary tests failed in 3 cases, of which there was one partial mole, one non-molar gestation, and one a mole of unclear type.

\section{Conclusion:}

- Genotyping appears to be the more reliable method, with failure in a smaller percentage of cases. Method failure was a result of a lack of clean, non-contaminated, material.

- Careful attention to dissection is a critical lab step which should be carefully evaluated in lab quality control. This step can also be improved by more advanced methods such as laser capture microdissection.

The majority of POCs could be classified using both methods.

There was no clear relationship between method failure and final diagnosis.

\begin{tabular}{|l|l|}
\hline Total nr of cases: & 72 \\
\hline Fianl diagnosis & 42 Partial mole (PM) \\
\hline & 14 Complete mole (CM) \\
\hline & 14 Non-molar (NML) \\
\hline \multicolumn{1}{|c|}{ Ploidy analysis } \\
\hline Successful & $50 / 72$ \\
\hline \multicolumn{2}{|c|}{$12 \mathrm{PM}, 7 \mathrm{CM}, 2 \mathrm{NML}$} \\
\hline \multicolumn{1}{|c|}{ STR Genotyping } & \\
\hline Successful & $60 / 72$ \\
\hline & $4 \mathrm{PM}, 1 \mathrm{CM}, 5 \mathrm{NML}$ \\
\hline
\end{tabular}

Table 1. Summary of results.

The combined analyses indicate that STR genotyping is a robust method, but pure villous and maternal material must be obtained. 